Изв. АН Эстонии, Физ. Матем., 1989, 38, № 4, 372-378

удК 539.3

A. ПУРО

\title{
РАЗДЕЛЕНИЕ УРАВНЕНИЙ ТЕОРИИ УПРУГОСТИ НЕОДНОРОДНЫХ ТЕЛ
}

\author{
(Представил Х. Абен)
}

Разделение уравнений теории упругости изотропного тела, основанное на выделении решения, описывающего деформацию кручения в плоскости или на сфере, полученное Гутманом $\left[{ }^{1}\right]$, в дальнейшем было обобщено на случай трансверсально $\left[{ }^{2}\right]$ и сферически трансверсально изотропных тел $\left[{ }^{3}\right]$. Для одномерной неоднородности такое разделение было выполнено в $\left[{ }^{4}\right]$, когда коэффициенты Ламе $\lambda(z), \mu(z)$ зависят от декартовой координаты, и в $\left[{ }^{5}\right]$, если $\lambda(r), \mu(r)-$ функции радиуса в сферической системе координат.

Симметрия трансверсально изотропных тел позволяет также проводить это разделение при указанных выше типах неоднородностей $\left[{ }^{6-8}\right]$.

Для неоднородной среды с постоянным коэффициентом жесткости (коэффициент Пуассона $v$ - произвольная дифференцируемая функция координат) также были предложены формы общего решения $\left[{ }^{9-11}\right]$.

Ниже разделение уравнений проводится, когда коэффициент жесткости является функцией декартовой координаты $z$, или радиуса $r$ в сферической системе координат, а коэффициент Пуассона - произвольная дифференцируемая функция координат. В случае трансверсальной изотропии вышеупомянутых типов такое разделение возможно, когда оба коэффициента сдвига являются функциями $z$ или $r$ для соответствующих типов изотропий. Относительно вектора массовых сил М делается предположение, что он выражается через потенциалы.

1. Трансверсально изотропное тело отнесено к прямоугольной декартовой системе координат. Ось $z$ направлена перпендикулярно плоскости изотропии тела.

Будем считать, что оба коэффициента сдвига $c_{44}=c^{-1},\left(c_{11}-c_{12}\right) / 2=$ $=G$ в обобщенном законе Гука

$$
\begin{array}{ll}
\sigma_{x x}=c_{11} \varepsilon_{x x}+c_{12} \varepsilon_{y y}+c_{13} \varepsilon_{z z}, & \sigma_{x y}=\left(c_{11}-c_{12}\right) \varepsilon_{x y}, \\
\sigma_{y y}=c_{12} \varepsilon_{x x}+c_{11} \varepsilon_{y y}+c_{13} \varepsilon_{z z}, & \sigma_{x z}=2 c_{44} \varepsilon_{x z}, \\
\sigma_{z z}=\sigma=c_{13}\left(\varepsilon_{x x}+\varepsilon_{x y}\right)+c_{33} \varepsilon_{z z}, & \sigma_{y z}=2 c_{44} \varepsilon_{y z}
\end{array}
$$

дифференцируемые функции только координаты $z$, а остальные коэффициенты упругости $c_{i k}$ - функции трех координат. Предполагается также, что вектор массовых сил $\boldsymbol{M}$ и вектор перемещений разлагаются на потенциальную и соленоидальную составляющие в плоскости изотропии и выражаются соответственно через потенциалы

$$
\begin{aligned}
& \mathbf{M}=\left(\frac{\partial \varphi}{\partial x}+\frac{\partial \Omega}{\partial y}\right) \mathbf{i}+\left(\frac{\partial \varphi}{\partial y}-\frac{\partial \Omega}{\partial x}\right) \mathbf{j}+\frac{\partial \chi}{\partial z} \mathbf{k} \\
& \mathbf{u}=\left(\frac{\partial F}{\partial x}+\frac{\partial N}{\partial y}\right) \mathbf{i}+\left(\frac{\partial F}{\partial y}-\frac{\partial N}{\partial x}\right) \mathbf{j}+w \mathbf{k}
\end{aligned}
$$


Подставляя выражение $\boldsymbol{M}$ и и в уравнениё равновесия $\operatorname{div} \sigma+\boldsymbol{M}=0$, получаем следующую систему трех уравнений

$$
\begin{gathered}
\frac{\partial}{\partial x}\left(Q_{1}+\varphi\right)+\frac{\partial}{\partial y}\left(Q_{2}+\Omega\right)=0, \quad \frac{\partial}{\partial y}\left(Q_{1}+\varphi\right)-\frac{\partial}{\partial x}\left(Q_{2}+\Omega\right)=0, \\
\frac{\partial}{\partial z}(\sigma+\chi)+\Delta_{+} \tau=0 .
\end{gathered}
$$

Здесь $Q_{1}=\frac{\partial \tau}{\partial z}+\frac{c_{13}}{c_{33}} \sigma+\frac{1}{d} \Delta_{+} F, \quad \Delta_{+}=\frac{\partial^{2}}{\partial x^{2}}+\frac{\partial^{2}}{\partial y^{2}}$,

$$
\begin{gathered}
Q_{2}=\left[G \Delta_{+}+\frac{\partial}{\partial z} c^{-1} \frac{\partial}{\partial z}\right] N, \quad d=\frac{c_{33}}{c_{33} c_{11}-c_{13}^{2}}, \\
\tau=c^{-1}\left(w+\frac{\partial}{\partial z} F\right), \\
\sigma_{z 2}=\sigma=c_{33} \frac{\partial w}{\partial z}+c_{13} \Delta_{+} F .
\end{gathered}
$$

Доказательство того, что система уравнений (1.1), (1.2) разлагается на уравнение $Q_{2}+\Omega=0$ относительно $N$ (потенциала нормального вращения), а также на систему из двух уравнений $Q_{1}+\varphi=0$ и (1.2)

$$
\begin{gathered}
\frac{\partial}{\partial z}\left[c_{44}\left(w+\frac{\partial F}{\partial z}\right)\right]+c_{13} \frac{\partial w}{\partial z}+c_{11} \Delta_{+} F=-\varphi \\
\Delta_{+}\left[c_{44}\left(w+\frac{\partial F}{\partial z}\right)\right]+\frac{\partial}{\partial z}\left[c_{33} \frac{\partial w}{\partial z}+c_{13} \Delta_{+} F\right]=\frac{-\partial \chi}{\partial z},
\end{gathered}
$$

(решение первого рода) идентично приведенному в $\left[{ }^{2,6,7}\right]$ и поэтому здесь приводиться не будет.

Аналогичное разделение уравнений возможно для динамических задач, если плотность среды является функцией только координаты $\boldsymbol{z}$. Приведем вывод других видов разрешающих уравнений первого рода. Для этого запишем уравнения (1.5), (1.6) в виде системы уравнений первого порядка

$$
\begin{gathered}
\frac{\partial \sigma}{\partial z}=-\Delta_{+} \tau-\frac{\partial \chi}{\partial z}, \\
\frac{\partial w}{\partial z}=\frac{\sigma}{c_{33}}-\frac{c_{13}}{c_{33}} \Delta_{+} F \\
\frac{\partial \tau}{\partial z}=-\frac{c_{13}}{c_{33}} \sigma-\frac{1}{d} \Delta_{+} F-\varphi \\
\frac{\partial F}{\partial z}=c \tau-w
\end{gathered}
$$

Удовлетворим уравнению (1.7), выражая $\sigma$ и $\tau$ через функцию $L$ (функцию напряжений)

$$
\sigma=\Delta_{+} L-\chi, \quad \tau=-\frac{\partial L}{\partial z} .
$$


Определив ш через $L$ и $F$ из (1.10) и подсставив $\sigma, \tau, w$ в систему (1.8), (1.9), получим

$$
\begin{gathered}
\frac{\partial^{2} F}{\partial z^{2}}+(c-b) \frac{\partial^{2} L}{\partial z^{2}}+c^{\prime} \frac{\partial L}{\partial z}+a \Delta_{+} L=a \chi-b \varphi, \\
\Delta_{+} F-d \frac{\partial^{2} L}{\partial z^{2}}+b \Delta_{+} L=b \chi-d \varphi .
\end{gathered}
$$

Здесь $w=-\left(c \frac{\partial L}{\partial z}+\frac{\partial F}{\partial z}\right), \quad b=d c_{13} c_{33}^{-1}$.

Система (1.11), (1.12) является смешанной в том смысле, что неизвестными являются функция напряжений $L$ и потенциал смещения $F$.

Рассмотрим более подробно случай однородных уравнений $\varphi=$ $=\chi=0$ (массовые силы отсутствуют).

В этом случае напряжения с учетом уравнений $(1.11)$, (1.12) выражаются формулами $\left(\Phi=\left[c_{11}-c_{12}\right] F\right)$

$$
\begin{gathered}
\sigma_{x x}=\frac{\partial^{2} L}{\partial z^{2}}-\frac{\partial^{2} \Phi}{\partial y^{2}}, \quad \sigma_{y y}=\frac{\partial^{2} L}{\partial z^{2}}-\frac{\partial^{2} \Phi}{\partial x^{2}}, \quad \sigma_{z z}=\Delta_{+} L \\
\sigma_{x y}=\frac{\partial^{2} \Phi}{\partial x \partial y}, \quad \sigma_{x z}=-\frac{\partial^{2} L}{\partial x \partial z}, \quad \sigma_{y z}=-\frac{\partial^{2} L}{\partial y \partial z}
\end{gathered}
$$

Из (1.13) следует $\left[{ }^{10}\right]$, что $L$ и $Ф$ являются диагональными элементами тензора функции напряжений $\psi$, в то время как остальные его элементы равны нулю $\psi=\operatorname{diag}\{L, L,-\Phi\}$. Таким образом, уравнения $(1.11),(1.12)$ можно получить из уравнений совместности для тензора напряжений, выраженного по формулам (1.13).

Уравнение относительно $L$ получим, исключая Ф из (1.11),

$$
\Delta_{+}\left(a \Delta_{+}-b \frac{\partial^{2}}{\partial z^{2}}+\frac{\partial}{\partial z} c \frac{\partial}{\partial z}\right) L+\frac{\partial^{2}}{\partial z^{2}}\left(d \frac{\partial^{2}}{\partial z^{2}}-b \Delta_{+}\right) L=0 .
$$

Здесь $a=d c_{11} c_{33}^{-1}$.

По форме оно совпадает с приведенным ранее $\left[{ }^{6,7}\right]$ и отличается тем, что коэффициенты $a, b, d$ могут зависеть от трех координат. Если $L$ и коэффициенты уравнения (1.14) не зависят от $x$ или $y$, то приходим к плоской задаче. Из формул (1.13), (1.14) видно, что $L$ переходит в функцию Эйри.

В заключение запишем систему уравнений (1.11), (1.12) в симметричном виде, используя замену $F=F_{0}-c L / 2$

$$
2 \Delta_{i} F_{0}=\mp \sqrt{D} \Delta_{i} L+c^{\prime \prime} L, \quad \begin{aligned}
& \text { при } i=1 \text { знак минус, } \\
& \text { при } i=2 \text { знак плюс. }
\end{aligned}
$$

Здесь $\quad \Delta_{i} F_{0}=\left(m_{i}^{2} \Delta_{+}+\frac{\partial^{2}}{\partial z^{2}}\right) F_{0}, \quad i=1,2$,

$m_{1,2}^{2}=[(c-2 b) \pm \sqrt{D}] /(2 d)-$ корни характеристического уравнения $d m^{4}-(c-2 b) m^{2}+a=0 ; \quad D=(c-2 b)^{2}-4 a d-\quad$ дискриминант этого уравнения.

2. Приведем основные разрешающие уравнения для случая изотропного тела. Предполагаем, что коэффицеинт сдвига $\mu$ это функция от $z$, а коэффициент Пуассона $v-$ функция трех координат. 
В этом случае система (1.11), (1.12) преобразуется к вйду

$$
\begin{gathered}
\frac{\partial^{2} F}{\partial z^{2}}+\frac{(2+v)}{2 \mu} \frac{\partial^{2} L}{\partial z^{2}}+\left(\frac{1}{\mu}\right)^{\prime} \frac{\partial L}{\partial z}+\frac{1-v}{2 \mu} \Delta_{+} L=\frac{1-v}{2 \mu} \chi-\frac{v}{2 \mu} \varphi, \\
\Delta_{+} F-\frac{1-v}{2 \mu} \frac{\partial^{2} L}{\partial z^{2}}+\frac{v}{2 \mu} \Delta_{+} L=\frac{v}{2 \mu} \chi-\frac{1-v}{2 \mu} \varphi .
\end{gathered}
$$

Рассмотрим запись разрешающих уравнений в переменных $F_{0}, L$ (вводим $\left.c(z)=[\mu(z)]^{-1}\right)$

$$
F_{0}=F+c L / 2 \text {. }
$$

В случае изотропного пространства дискриминант $D=0$, корни характеристического уравнения равны $m_{1}{ }^{2}=m_{2}{ }^{2}=1$ и уравнения (1.15) совпадают

$$
2 \Delta F_{0}=c^{\prime \prime} L-c(\varphi-\chi) .
$$

В качестве второго разрешающего уравнения можно выбрать одно из системы (2.1), (2.2).

В частности, выбираем (2.2) и записываем его в переменных $F_{0}, L$

$$
c(1-v) \Delta L=2 \Delta_{+} F_{0}-c[v \chi-(1-v) \varphi] .
$$

Необходимо отметить, что функции $F_{0}, L$ использовались ранее А. Е. Лявом для вывода общего решения осесимметричной деформации тела через одну бигармоническую функцию - функцию Лява.

В дальнейших исследованиях они не нашли широкого применения. Как видно из (1.15) использование этих функций облегчает нахождение и исследование решений для трансверсально изотропных тел.

Подействуем оператором $\Delta$ на $(2.5)$ и подставим значение $\Delta F_{0}$ из (2.4), в результате получим уравнение относительно

$$
\Delta[c(1-v) \Delta L]-c^{\prime \prime} \Delta_{+} L=\Delta[c(1-v) \varphi-c v \chi]+\Delta_{+}[c(\chi-\varphi)] .
$$

В отсутствие массовых сил это уравнение по форме совпадает с уравнением, введенным В. П. Плевако [ $\left.{ }^{4}\right]$

$$
\Delta[c(1-v) \Delta L]-c^{\prime \prime} \Delta_{+} L=0 .
$$

Физический смысл уравнения (2.6) виден из записи в форме

$$
2 \Delta\left[(1-v)(1-2 v)^{-1} \operatorname{div} \mathbf{u}\right]=c^{\prime \prime} \sigma .
$$

Здесь учтено, что $\Delta L$ можно выразить

$$
\Delta L=2 \mu(1-2 v)^{-1} \operatorname{div} \mathbf{u} .
$$

Уравнение (2.7) является разрешающим [ $\left.{ }^{9-11}\right]$ при построении общего решения в случае $\mu=$ const при помощи гармонического вектора и вспомогательной функции.

При $c^{\prime \prime} \neq 0$ можно в качестве разрешающей функции использовать $F_{0}$. Для этого выразим $L=\left(c^{\prime \prime}\right)^{-1}\left[2 \Delta F_{0}+c(\chi-\varphi)\right]$ из (2.4) и, подставляя $L$ в (2.5), получим уравнение относительно $F_{0}$

$$
c(1-v) \Delta\left\{\left(c^{\prime \prime}\right)^{-1}\left[2 \Delta F_{0}+c(\chi-\varphi)\right]\right\}=2 \Delta_{+} F_{0}+c[v \chi-(1-v) \varphi] .
$$

Если массовые силы отсутствуют, уравнение упрощается

$$
c(1-v) \Delta\left[\left(c^{\prime \prime}\right)^{-1} \Delta F_{0}\right]=\Delta_{+} F_{0} .
$$

Перемещения в переменных $F_{0}, L$ соответственно равны

$$
u_{x, y}=\frac{\partial}{\partial x, y}\left[F_{0}-\frac{L}{2 \mu}\right] ; \quad w=-\left[\frac{\partial F_{0}}{\partial z}+\frac{1}{2 \mu^{2}} \frac{\partial}{\partial z}(\mu L)\right] .
$$


Н̈апряження однородных уравнений через функции $L$ и $\Phi_{0}=2 \mu F_{0}$ выражаются формулами

$\begin{array}{ll}\sigma_{x x}=\frac{\partial^{2} L}{\partial z^{2}}+\frac{\partial^{2}\left(L-\Phi_{0}\right)}{\partial y^{2}}, \quad \sigma_{x z}=-\frac{\partial^{2} L}{\partial x \partial z}, & \sigma_{z z}=\Delta_{+} L, \\ \sigma_{y y}=\frac{\partial^{2} L}{\partial z^{2}}+\frac{\partial^{2}\left(L-\Phi_{0}\right)}{\partial x^{2}}, & \sigma_{y z}=-\frac{\partial^{2} L}{\partial y \partial z}, \quad \sigma_{x y}=\frac{\partial^{2}}{\partial x \partial y}\left(\Phi_{0}-L\right) .\end{array}$

3. Если в выражении тензора напряжений $\sigma$ сферически трансверсально изотропной среды

$$
\begin{array}{ll}
\sigma_{r r}=c_{11} \varepsilon_{r r}+c_{12}\left(\varepsilon_{\theta \theta}+\varepsilon_{\varphi \varphi}\right), & \sigma_{\varphi \varphi}=c_{12} \varepsilon_{r r}+c_{22} \varepsilon_{\varphi \varphi}+c_{23} \varepsilon_{\theta \theta}, \\
\sigma_{\theta \theta}=c_{12} \varepsilon_{r r}+c_{22} \varepsilon_{\theta \theta}+c_{23} \varepsilon_{\varphi \varphi}, & \sigma_{r \varphi}=2 c_{44} \varepsilon_{r \varphi}, \\
\sigma_{r \theta}=2 c_{44} \varepsilon_{r \theta}, & \sigma_{\theta \varphi}=\left(c_{22}-c_{23}\right) \varepsilon_{\theta \varphi},
\end{array}
$$

оба коэффициента сдвига $c_{44}, 2 G=c_{22}-c_{23}$ функции только координаты $r$ (остальные коэффициенты могут зависеть от трех переменных), то уравнения равновесия $\operatorname{div} \sigma=0$ разделяются на уравнение, описывающее нормальное вращение (уравнение второго рода) и систему двух уравнений (решение первого рода). Ранее приведенный вывод $\left[{ }^{5,6,8}\right]$ без изменений переносится на этот более общий случай, причем полученные формулы $\left[{ }^{8}\right]$ полностью сохраняют свой вид.

Приведем разрешающие уравнения первого рода относительно wсоставляющей вектора $u_{r}$ и потенциала $F$

$$
\begin{gathered}
\bar{D} \sigma+\Delta_{*} \tau=2\left\{\frac{c_{12}}{r} \partial+\frac{c_{22}+c_{23}}{r^{2}}\right\} w+\frac{c_{22}+c_{23}}{r} \Delta_{*} F \\
\bar{D}\left(\frac{\tau}{r}\right)+\frac{\tau}{r^{2}}=-\left\{\frac{c_{12}}{r} \partial+\frac{c_{22}+c_{23}}{r^{2}}\right\} w-\left(\frac{c_{22}-c_{23}}{r^{2}}+\frac{c_{22}}{r} \Delta_{*}\right) F .
\end{gathered}
$$

Здесь $\partial v=\frac{\partial}{\partial r} v, \quad \bar{D} v=\frac{1}{r^{2}} \frac{\partial}{\partial r}\left(r^{2} v\right)$,

$\Delta_{*} v=\frac{1}{r^{2}}\left[\frac{1}{\sin \theta} \frac{\partial}{\partial \theta}\left(\sin \theta \frac{\partial}{\partial \theta} v\right)+\frac{1}{\sin ^{2} \theta} \frac{\partial^{2} v}{\partial \varphi^{2}}\right]-$ оператор Бельтрами,

$$
\begin{gathered}
\sigma=c_{11} \partial w+c_{12}\left[\frac{2 w}{r}+\Delta_{\star} F\right], \\
\tau=c_{44}\left[w+r^{2} \partial\left(\frac{F}{r^{2}}\right)\right] .
\end{gathered}
$$

Уравнения (3.1), (3.2) являются уравнениями равновесия, а (3.3), (3.4) уравнениями закона Гука для $\sigma_{r r}=\sigma$ и $\tau$-потенциала компонент тензоров

$$
\sigma_{r \theta}=\frac{1}{r} \frac{\partial}{\partial \theta} \tau=D_{\theta} \tau, \quad \sigma_{r \varphi}=\frac{1}{r \sin \theta} \frac{\partial}{\partial \varphi} \tau=D_{\varphi} \tau .
$$

Причем компоненты смещения $u_{\theta}=D_{\theta} F, u_{\Phi}=D_{\varphi} F$. Сложив (3.1) с удвоенным (3.2), получаем уравнение

$$
\bar{D}\left(\sigma+\frac{2 \tau}{r}\right)+\left(\Delta_{*}+\frac{2}{r}\right)\left(\tau+\frac{c_{22}-c_{23}}{r} F\right)=0,
$$

решение которого можно выразить через $L$, заменив

$$
\sigma=\left(\Delta_{*}+\frac{2}{r^{2}}\right) L-\frac{2 \tau}{r}, \quad \tau=-\partial L-\frac{\left(c_{22}-c_{23}\right)}{r} F .
$$


Тем самым получй систему трех уравнений: уравнения равновесия (3.2) и закона Гука $(3.3),(3.4)$ относительно $L, F, w$. Выражая w из (3.4) через $L$ и $F$

$$
w=-\frac{1}{c_{44}}\left[\partial L+c_{44} \partial F+\frac{c_{22}-c_{23}-2 c_{44}}{r} F\right]
$$

и подставляя в (3.3) и (3.2), получаем систему уравнений относительно $L$ и $F$, аналогичную (1.11), (1.12).

Из формул для напряжения $\left(\Phi=\left[c_{23}-c_{22}\right] F, \tau=\frac{\Phi}{r}+\partial L\right)$

$$
\begin{gathered}
\sigma=\frac{2}{r^{2}} \Phi-\left[\Delta_{*}+\frac{2}{r} D\right] L, \quad \sigma_{r \theta}=D_{\theta} \tau, \quad \sigma_{r \varphi}=D_{\varphi} \tau \\
\sigma_{\theta \varphi}=-D_{\theta} D_{\varphi} \Phi, \quad \sigma_{\varphi \varphi}=\left[D_{\theta}^{2}-\frac{1}{r} \partial\right] \Phi-D^{2} L \\
\sigma_{\theta \theta}=\left[D_{\varphi}^{2}-\frac{1}{r} \partial+\frac{\operatorname{ctg} \theta}{r} D_{\theta}\right] \Phi-D^{2} L
\end{gathered}
$$

следует, что $\Phi$ и $L$ являются компонентами тензора функции напряжений $\psi_{r r}=\Phi, \psi_{\varphi \varphi}=\psi_{\theta \theta}=-L$, у которого недиагональные члены равны нулю.

Рассмотрим более подробно изотропное тело. Разрешающая система уравнений

$$
\begin{gathered}
\Delta\left(\frac{L}{2 \mu r^{2}}\right)+\frac{L}{r^{2}}\left[\frac{1}{r} \partial\left(\frac{1}{\mu}\right)-\frac{1}{2} \partial^{2}\left(\frac{1}{\mu}\right)\right]+\Delta\left(\frac{F}{r^{2}}\right)-\frac{F}{\mu r^{3}} \partial \mu=0 \\
\bar{D}(\partial L)-\frac{v}{1-v} \Delta_{*} L-\frac{2 \mu}{1-v} \Delta_{*} F=\frac{-2}{(1-v) r}(\partial L+2 \mu \partial F)+ \\
+\frac{2 v}{(1-v) r^{2}}(L+2 \mu F)-\frac{2}{r} F \partial \mu
\end{gathered}
$$

заменой переменной $2 \mu F=2 \mu F_{0}-L$ сводится к

$$
\begin{gathered}
\Delta\left(\frac{F_{0}}{r^{2}}\right)=\frac{L}{2 r} \partial\left[\frac{1}{r} \partial\left(\frac{1}{\mu}\right)\right]-\frac{F_{0}}{\mu r^{3}} \partial \mu \\
\Delta L-\frac{2 \mu}{1-v}\left(\Delta_{*} F_{0}-\frac{2}{r} \partial F_{0}+\frac{2 v}{r^{2}} F_{0}\right)=\frac{(1+v) L}{\mu r(v-1)} \partial \mu-\frac{2 F_{0}}{r} \partial \mu .
\end{gathered}
$$

Уравнения (3.8), (3.9) переходят в (2.4), (2.5) при $r \rightarrow \infty$. Коэффициент при $L$ в (3.8) обращается в ноль, если $\mu=\left(c r^{2}+c_{1}\right)^{-1}$, в частности, при $\mu=$ const $\left(F_{0} / r\right)^{2}-$ гармоническая, а $L-$ бигармоническая функции. Если коэффициент при $L$ в (3.8) неравен нулю, то систему можно свести к одному уравнению относительно $F_{0}$, аналогичному $(2.8)$. рода

В заключение приведем выражение смещений решения первого

$$
u_{\theta, \varphi}=D_{\theta, \varphi}\left[F_{0}-\frac{L}{2 \mu}\right], \quad w=-\left[\partial F_{0}+\frac{1}{2 \mu^{2}} \partial(\mu L)\right] .
$$

Таким образом, для среды с рассмотренными выше неоднородностями решение выражается через две функции (решение первого и второго рода), которые удовлетворяют уравнениям в частных производных второго и четвертого порядков. 
1. Гутман С. Г. Изв. ВНИИ гидротех., $1948,37,89-101$.

2. Hu Hai-chang // Acta Sci. Sinica, 1953, 11, № 2, 145-151.

3. Hu Hai-chang // Acta Sci. Sinica, 1954, 12, № 3, 247-260.

4. Плевако В. П. // Прикл. мат. и мех., 35, вып. 1, 853-860.

5. Пуро А. Э. // Прикл. мат. и мех., 1974, 38, вып. 6, 1139-1144.

6. Пуро А. Э. // Канд. дис., Таллинн, 1975.

7. Раппопорт Р. М. // Прикл. мат. и мех., 1976, 40, вып. 5, 956-958.

8. Пуро А. Э. // Прикл. механика, 1980, I6, № 2, 40-44.

9. Boudet, $R$. // Comptes rendus hebdomadaires des séances de l'Académie des Sciences, Ser. A, 1966, 263, № 19, 692-694.

10. Ломакин B. А. Теория упругости и однородных тел. М., Изд-во Моск. ун-та, 1976.

11. Бородачев А. Н. // Прикл. мат. и мех., 1987, 51, вып. 4, 611-615.

Калининградский технический институт рыбной промышленности и хозяйства
Поступила в редакцию $24 / \mathrm{X} 1988$

\section{A. PURO}

\section{MITTEHOMOGEENSETE KEHADE ELASTSUSTEOORIA VORRANDITE ERALDAMINE}

Võrrandid on eraldatud juhul, kui elastsuskoefitsient on ristkoordinaadi $z$ või sfäärilise koordinaadi $r$ funktsioon ning Poissoni koefitsient sōltub kōigist kolmest koordinaadist. Kehade transversaalse isotroopia korral on vōrrandite eraldamine vōimalik, kui mõlemad elastsuskoefitsiendid on $z$-i või $r$-i funktsioonid. On eeldatud, et massijōudude vektoril on potentsiaal.

\section{A. PURO}

\section{SEPARATION OF EQUATIONS OF NON-HOMOGENEOUS BODIES}

The separation is carried out in cases when the coefficient of shear depends on the Decart coordinate $z$ or radius $r$ of the spherical system coordinates.

An analogical separation happens in the transversal isotropic body, when both coefficients of shear depend on $z$ or $r$ for the corresponding isotropy. It is presumed that mass forces have the potential. 